\title{
The coldest place in Ireland
}

\author{
Laura Russell
}

Physics Department, UCC

\section{Introduction}

Did you know that the Quantum Optics Laboratory in Tyndall National Institute is the coldest place in Ireland? The physicists in this laboratory use lasers to push atoms in such a way as to slow them down. When an atom gets slowed down, its temperature also decreases. This technique is called laser cooling and was first demonstrated in 1978 by Wineland, Drullinger and Walls.

Physicists use the Kelvin (K) scale to measure temperatures which is an extension of the degree Celsius scale down to absolute zero, the lowest temperature that can theoretically be reached. Absolute zero Kelvin represents a complete absence of heat energy. While we may have thought our weather couldn't get much colder last winter here in Ireland, cold atoms are unimaginably chilled, at nearly absolute zero Kelvin, or $-273{ }^{\circ}$ Celsius.

\section{Laser cooling and magnetic trapping}

The temperature of an atom is related to how fast it's moving. The air we are breathing is comprised of atoms moving around at 600 miles per hour; they are full of energy and have a temperature of $300 \mathrm{~K}$ or, what we term "room temperature." A cold atom moves around very slowly. If we can slow the atom down, its temperature will be reduced. To laser cool an atom which is moving left to right, we shine a laser beam against it from right to left. When the laser beam is just the right colour or wavelength, the beam will bounce off the atom with more energy than it hit the atom with. This extra portion of energy has been taken away from the atom and therefore the atom is just a little colder than before it encountered the laser beam. If the laser beam is the wrong wavelength, it will pass straight through the atom and have no effect. The science of keeping lasers at precisely one colour or wavelength is a fascinating and challenging research area.

As we live in a 3-dimensional world, we need to slow the atom in all three directions. Therefore we send six laser beams towards it - top and bottom, left and right, front and back. While the six lasers push the cold atoms to the intersection point of the beams, we must also use a magnetic field to keep the atoms in a tightly confined position. The magnetic field we use would look like an egg-cup if we could see it with our eyes. Our 
laser-cooled atoms fall into the bottom of the magnetic "cup". Combined, the six laser beams and the magnetic field are known as a magneto-optical trap, or MOT — see figure 1.

The MOT is arranged inside an ultra-high vacuum chamber. This is a large stainless steel compartment that has as much air pumped out from it as possible. This means that very few non-laser-cooled atoms will interfere with the laser-cooled ones at the centre of the MOT. Inside the vacuum chamber, the pressure is $10^{-10} \mathrm{mbar}$, similar to that in Deep Space. When the MOT is switched on, a small cloud, measuring 1 millimetre in width and containing 10 million atoms with a temperature of approximately 100 micro-Kelvin forms at the centre. The photograph in figure 2 is taken with a CCD camera pointing through a glass window in the vacuum chamber. The laser beam intersection point is clearly visible, and at its centre is a cloud of cold atoms.

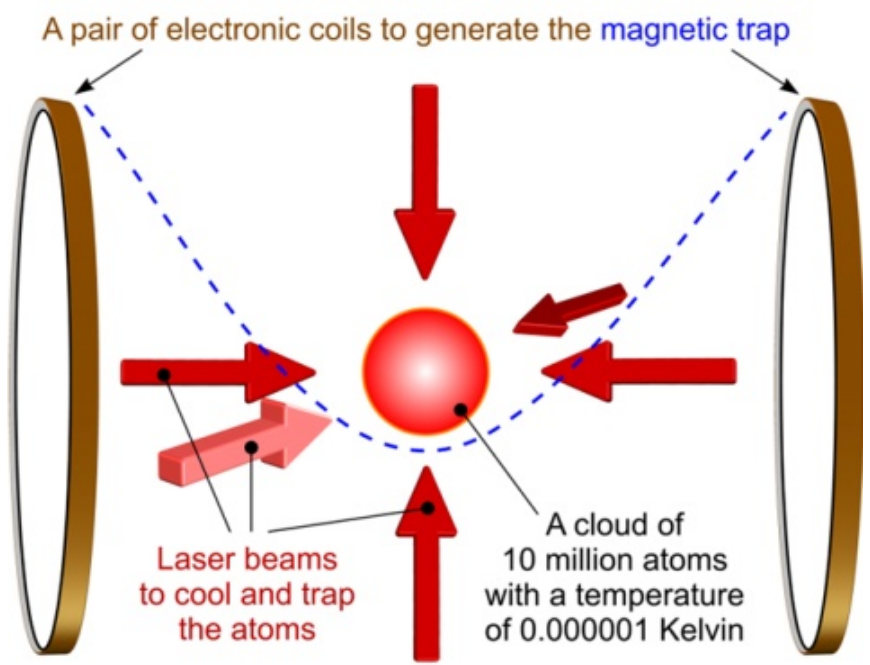

Figure 1: Schematic illustration of a magneto-optical trap (MOT). The six laser beams cool the atoms and push them to the intersection point while the magnetic trap (generated with a pair of electronic coils) confines the atoms in a small volume approximately $1 \mathrm{~m}$

\section{My Research}

My own research is a step along the way towards a quantum computer. In the Quantum Optics Group laboratory, I investigate what happens when you move the cloud of cold rubidium atoms near a glass surface. Rubidium is a silvery metal that looks a lot like aluminium. It is released as a gas into the vacuum chamber and laser cooled with infrared laser light. This means the laser beam is invisible to the naked eye but can be viewed with a CCD camera.

To test the atoms near a glass surface, a very narrow glass rod called an optical nanofibre is placed inside the vacuum chamber and the cold rubidium atoms are positioned around 


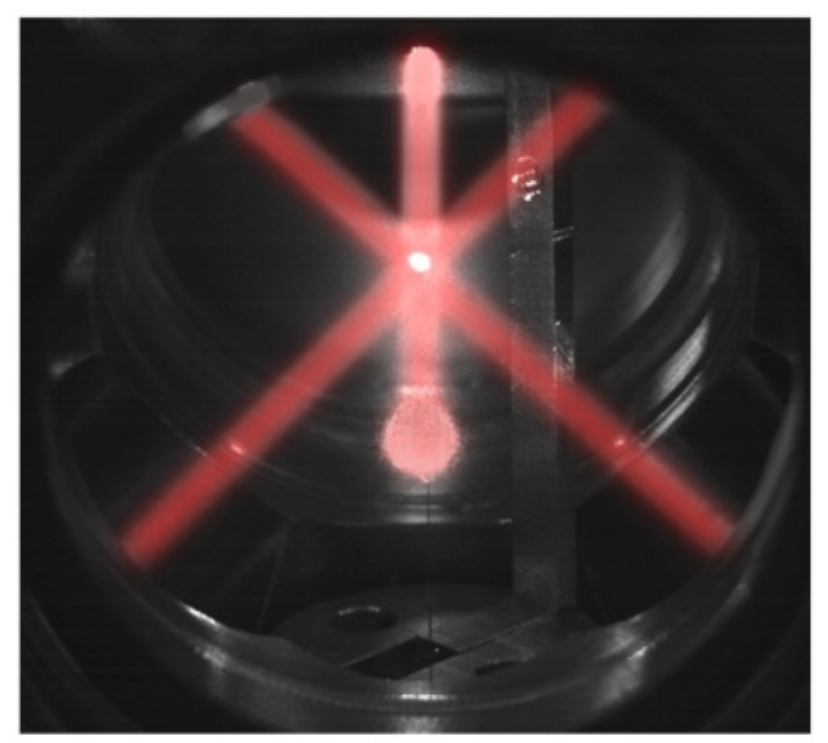

Figure 2: A CCD image of a cold cloud of rubidium atoms which have been laser cooled by the red laser beams to temperatures of a millionth of a Kelvin. The white fluorescent cloud forms at the intersection of the beams.

it. The nanofibre is 100 times smaller than a human hair and is fabricated from what is now an everyday tool in connectivity - optical fibre. To make this tiny device, a section of optical fibre is heated and stretched apart until it is only 600 nanometres in diameter. Figure 3 shows a CCD image of the rubidium cloud surrounding the nanofibre - the nanofibre appears larger than it actually is because of the laser light reflecting off it. The cloud of atoms, which is fluorescing while being laser cooled, is slightly elongated because gravity is pulling it downwards. The right hand image (figure 4) models what the system looks like in greater detail. The cloud of rubidium atoms fluoresces and a portion of this light leaks into the nanofibre and travels towards a detector.

As the atoms are so small, they will see the nanofibre as a large glass surface. In fact, each atom can see an image of itself in the glass. An atom is made up of positive and negative

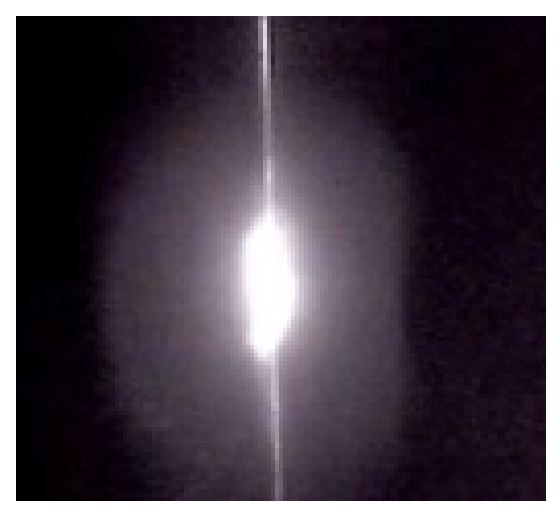

Figure 3: A CCD image of the cold cloud of rubidium atoms surrounding an optical nanofibre. The cloud is elongated because of gravity and the nanofibre appears larger than it actually is due to the intensity of the light reflecting off it. 


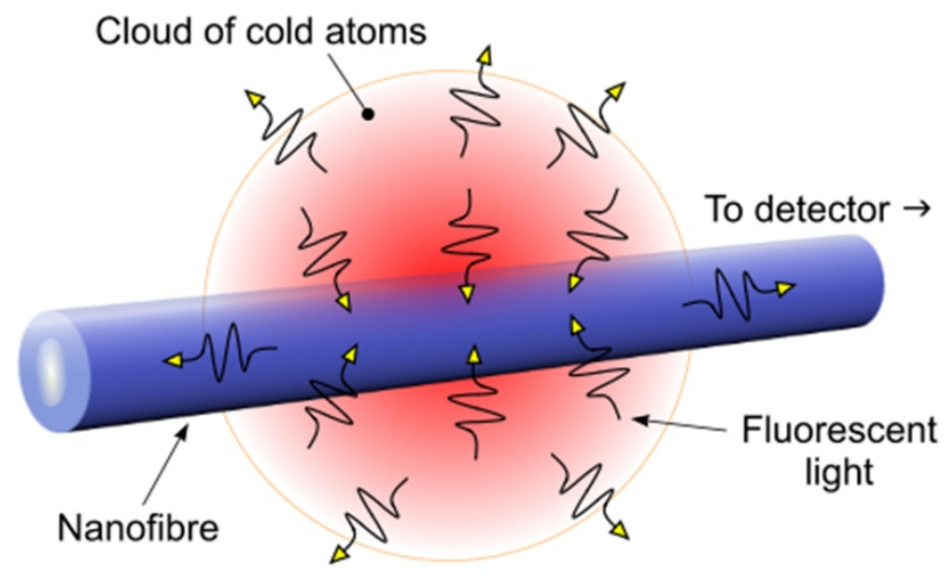

Figure 4: A schematic illustration of the rubidium cloud and nanofibre system. The fluorescent light escapes from the cloud in all directions but a portion of it can enter the nanofibre and travel towards a detector.

charges that are moving around constantly. Every now and again, there will be mostly positive charges on one side of the atom and negative charges on the other side. In the mirror image of the atom in the glass, these charges are reversed and, as the old saying goes: opposites attract! The positive charges on the atom will be attracted to the negative charges on its reflection and vice versa. It's almost as if the atom is attracted to its own reflection. This is called the van der Waals effect - which is the mechanism responsible for geckos sticking to walls. While useful for geckos, this is a major concern for quantum devices of the future where cold atoms will be positioned near surfaces in order to be probed. If many atoms stick to the surface the efficiency of the device will be destroyed. With my experiment, the nanofibre also acts as a detector for the atoms so that we can learn how close an atom is to surface and even control this distance.

\section{Using the nanofibre as a detector}

Figure 5 displays a fundamental result from my research. The magnetic trap is switched on and off to allow the cold atoms to gather around the nanofibre and then disperse, respectively. The sudden increase in the signal is from the light from the cold atoms entering the nanofibre and travelling towards a detector via the normal sections of unstretched optical fibre.

\section{Spectroscopy with cold atoms and nanofibres}

The optical nanofibre is created from standard optical fibre which, for example, is used to carry light signals for broadband connectivity — this means that we can launch light through one end of the fibre and it will travel all the way to the other end even through the narrow section of nanofibre. Figure 6 shows a schematic image similar to that in 


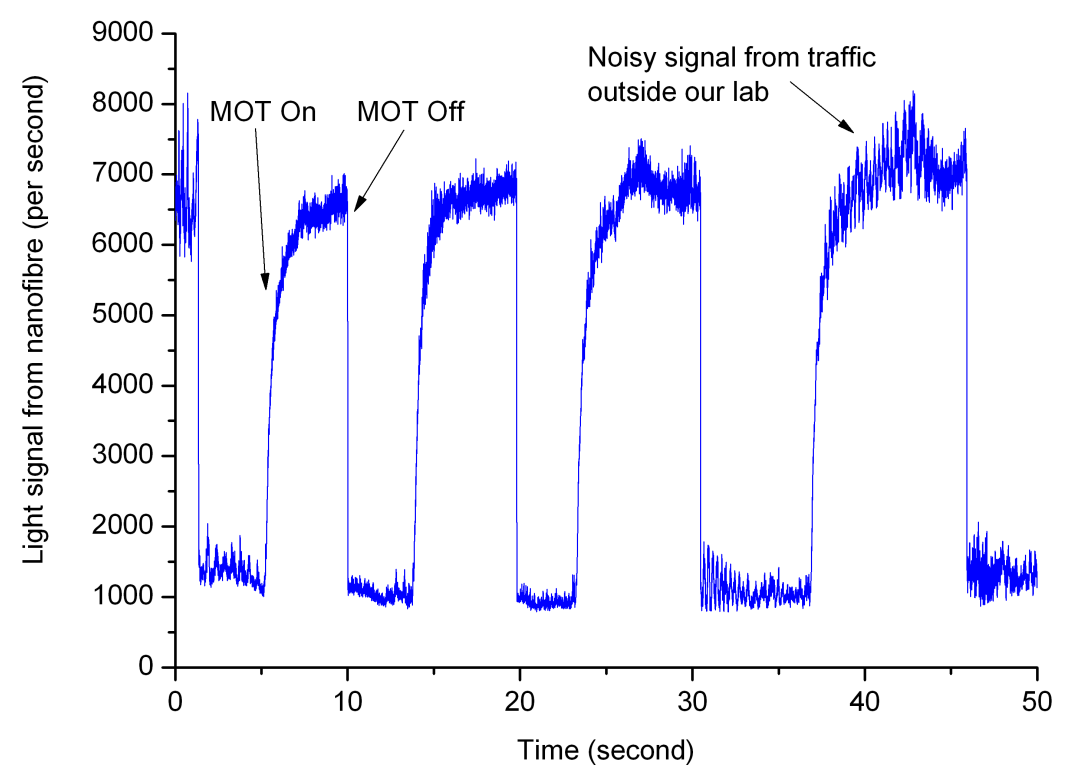

Figure 5: Each time the MOT is switched on the cloud of atoms gathers around the nanofibre and fluoresces into it. This plot shows what the detector observes at one end of the nanofibre - a large increase in light coming from the nanofibre each time the MOT is activated. The rubidium cloud is extremely sensitive to external fluctuations. If there is heavy traffic on the road outside our laboratory, we observe a fluctuating signal as shown in the last peak in the figure.

figure 4 with the addition of a probe beam sent through the nanofibre. This probe beam leaks out of the nanofibre and is absorbed by the atoms provided the beam is the correct wavelength. This means that the amount of light after the cloud will have decreased and we can examine this decrease or absorbance with a detector. This type of experiment is called spectroscopy and is used by scientists all over the world to gain characteristic information about samples of material, whether it is gas, liquid or solid.

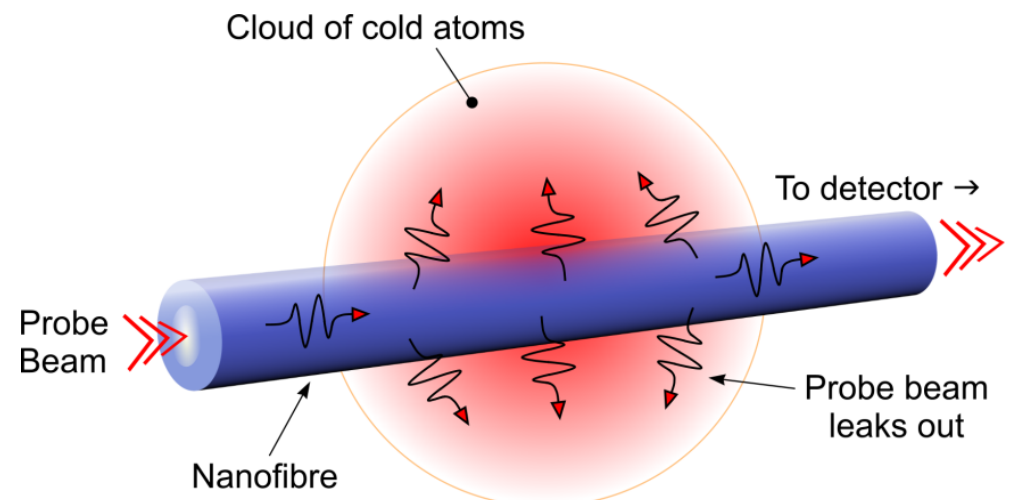

Figure 6: By sending a probe beam through the nanofibre, it leaks out and interacts with the surrounding cold atoms. This system allows us to perform spectroscopy on the cold cloud of rubidium. 


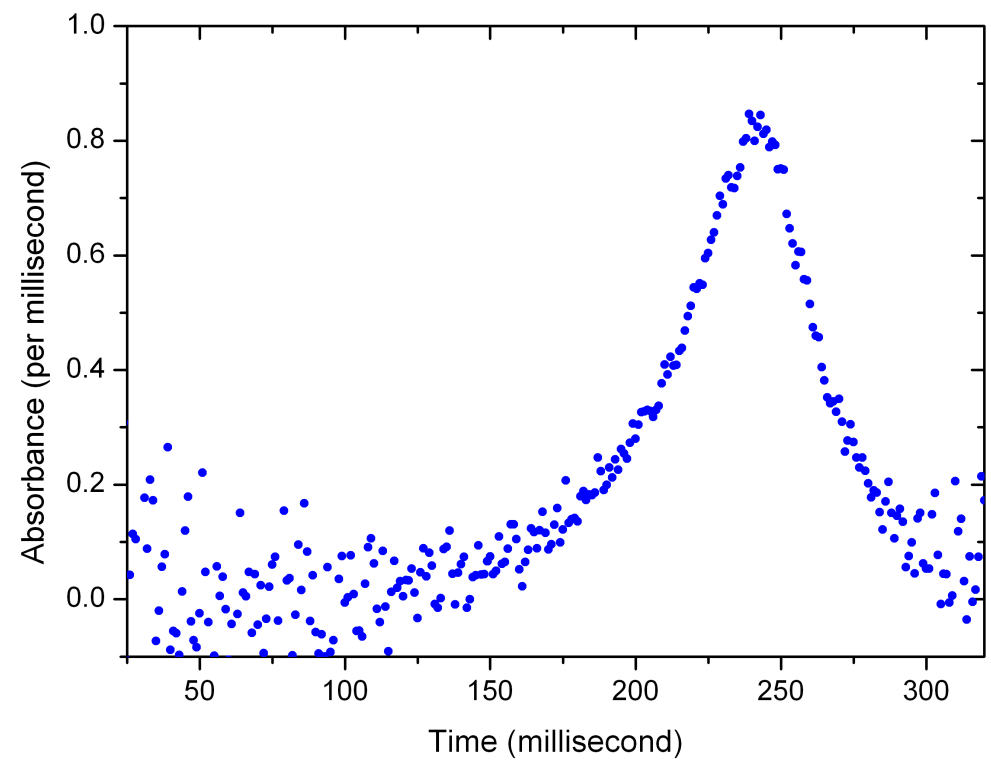

Figure 7: An absorbance profile for rubidium in a MOT. During these 300 milliseconds, the probe beam wavelength (colour) is scanned over a very small range. When it is the correct wavelength, the rubidium atoms in the MOT absorb as much of it as possible - this corresponds to the highest point in the graph where almost 0.9 absorbance is reached. The absorbance is much lower further away from this point because the atoms do not absorb the beam if it is the wrong wavelength.

Figure 7 displays a typical absorbance profile for cold rubidium atoms in a MOT. During this 300 millisecond timeframe, the wavelength of the probe beam is scanned over a very small range. When the wavelength of the beam is correct, the atoms will absorb as much of it as possible - this is the point on the graph where it reaches almost 0.9 , or $90 \%$, of the full absorbance. Away from this point, the beam is not absorbed as much.

\section{Outlook}

Despite sounding quite alien, cold atoms have many uses in some of today's essential technologies. For example, the world's most precise atomic clock, which uses cold strontium atoms, neither gains nor loses a second in more than 200 million years. In each of the 24 GPS satellites orbiting our Earth, there are four atomic clocks. These satellites are used by everyone from the military to the taxi driver. An error of just one nanosecond (or billionth of a second) in the GPS clock translates to an error of one foot on Earth. This may not seem like much, but is critical if you are relying on a GPS to land on an aircraft carrier, or synchronise signals from radio telescopes in astrophysics.

In the future, quantum computing devices will rely on scientists harnessing quantum behaviour to create systems that can far exceed the speed and processing capabilities of 
current silicon-based computers. Cold atoms will play a central role in this because quantum computers will require us to individual probe single cold atoms in order to encode them with a " 0 " or a "1" to create a binary system which is what modern computers use to perform computational tasks. With this research and the efforts of other research groups worldwide, the iQuantumPhone is not far away!

I wish to thank my supervisor, Dr. Síle Nic Chormaic and my colleagues from the Quantum Optics Group. This work is partially supported by Science Foundation Ireland under Grant Nos. 07/RFP/PHYF518 and 08/ERA/I1761 through the NanoSci-E+ Project NOls, and the Higher Education Authority via the INSPIRE programme. I acknowledge support from IRCSET under the Embark Initiative. 